\title{
Correspondence
}

\section{Creating Expertise in Health and Healing}

To the Editor: Dr. Whitcomb's ${ }^{1}$ article on the redesign of residency training in family medicine was read with great interest. It was refreshing to have someone of his insight and expertise give a view of our specialty from the outside. His comments will help facilitate needed educational change to better serve patient need.

I would like to take his recommendations and repackage them into a definition that retains the heart of our specialty and allows us to be successful in recruiting future colleagues. Dr. Whitcomb recommends that we focus family medicine training to create chronic disease experts to serve the rising prevalence of these conditions in our culture. This supports a diseaseoriented focus and does not honor the relationshipcentered care that allows insight into understanding how to prevent or reverse the disease process. Being a "chronic disease specialist" feels heavy, burdensome, and reductionistic. Not once have I had a medical student come up to me and say, "I want to be a chronic disease specialist!" But I am seeing my younger colleagues have a passion to understand how the body self-heals. Their pupils dilate when they talk about being physician healers. This defines the package of what it means to be a family doc. Family medicine was founded on the bio-psycho-social model and the specialty is in the best position to use this insight to create experts in health and healing. This is what students want to be "experts" in. They want to be navigators of health, not a slave to the markers of chronic disease management. They want to develop meaningful relationships that allow them to reach in and grab onto the essence of what is needed for positive behavior change. They want to be experts in salutogenesis (the creation of health) as well as pathogenesis (the creation of disease and suffering).

Creating expertise in chronic disease supports a passive health care model in which our patients become more dependent on expensive external influences for health (pharmaceuticals, surgical therapy). Antidepressants and proton pump inhibitors are prescribed at a cost of billions of dollars each year. We simply treat the symptoms instead of asking the deeper question: Why is everyone in America depressed with an upset stomach? Family medicine has the opportunity to create leaders in understanding the process of healing so we can reverse the trend toward disease and empower healthy lifestyle behaviors. Success in defining this package will bring more joy to our work and reduce disease burden and the need for chronic disease experts.

David Rakel, MD

University of Wisconsin Department of Family Medicine, Madison

\section{Reference}

1. Whitcomb ME. Preparing the personal physician for practice $\left(\mathrm{P}^{4}\right)$ : meeting the needs of patients: redesign of residency training in family medicine. J Am Board Fam Med 2007;20(4):356-64.

doi: 10.3122/jabfm.2007.06.070165

\section{Family Physician Responds to the Future of Family Medicine}

To the Editor: I read with interest Dr. Green's ${ }^{1}$ commentary on the preparing the personal physician for practice initiative. It is necessary to go through the process of examining our specialty in light of the clear and increased need of most people to have a personal physician. It is also necessary to evaluate the strengths and weaknesses of our specialty in times of decreased attraction of our specialty to our brightest medical students. I believe this to be exactly the position of our mentors of the 1960s who "rescued" the field of general medicine in forming the specialty of family medicine.

As I review articles in the July-August issue of the journal, I note an apparent lack of evidence or input from those of us who spend almost all our professional time actually practicing family medicine. Evidence-based medicine and use of new computer technologies is certainly a present, needed change for my generation of family docs. Residency directors cannot truly expect to do more than train a new generation to grow with technology, with new medical evidence, and with their own observations. That training must include self-evaluation and learning by personal observation and should last a whole career!

This practicing family physician hopes that leaders of this project can devise and find support of trials of their ideas and ideals in actual practices. Enrolling experienced practitioners in serious discussions of the future of family medicine is critically necessary. Despite the struggles of practice, we do a needed and valuable service to the residents of our communities. Finding ways to give credit to our strengths while honestly seeking to correct our weaknesses- that has to be the goal of our specialty if it is to survive.

$$
\begin{array}{r}
\text { James W. Redka, MD } \\
\text { Susquehanna Health } \\
\text { Williamsport, PA }
\end{array}
$$

\section{Reference}

1. Green LA, Pugno P, Fetter GJ, Jones SM. Preparing the personal physician for practice $\left(\mathrm{P}^{4}\right)$ : a national program testing innovations in family medicine residencies. J Am Board Fam Med 2007;20(4):329-31.

doi: 10.3122/jabfm.2007.06.070171

The above letter was referred to the authors of the article in question, who offer the following reply. 